\title{
Fast determination of toxic diethylene glycol in toothpaste by ultra-performance liquid chromatography-time of flight mass spectrometry
}

\author{
Félix Hernández • María Ibáñez • Juan V. Sancho
}

Received: 11 January 2008 /Revised: 5 March 2008/Accepted: 19 March 2008/Published online: 15 April 2008

(C) Springer-Verlag 2008

\begin{abstract}
A rapid method for determining diethylene glycol (DEG) in toothpaste based on the use of ultraperformance liquid chromatography (UPLC) coupled to time-of-flight mass spectrometry (TOF-MS) has been developed. The method has been validated in toothpaste samples spiked at different levels, $0.005,0.1$ and 5\%, obtaining satisfactory recoveries (74-98\%) and relative standard deviations $(<4 \%)$. Quantification was carried out by using matrix-matched standards calibration. The developed method was applied to several types of toothpaste, making identification and quantification of DEG and other polyethylene glycols (PEG) feasible with very little sample manipulation, as only extraction with water is required. The excellent sensitivity of TOF-MS analysis performed in fullscan acquisition mode allowed the determination of DEG at concentration levels as low as $0.005 \%$ in samples and its reliable identification via the mass accuracy measurements provided by this instrument $(<5 \mathrm{ppm})$.
\end{abstract}

Keywords UPLC · TOF-MS · Toxic diethylene glycol · Toothpaste

\section{Introduction}

On May 2007, the US Food and Drug Administration (FDA) advised US pharmaceutical manufacturers to test all batches of glycerine, a sweetener commonly used worldwide in drug products, for the toxic chemical diethylene

F. Hernández $(\bowtie) \cdot M$. Ibáñez $\cdot$ J. V. Sancho

Research Institute for Pesticides and Water, University Jaume I,

12071 Castellón, Spain

e-mail: felix.hernandez@qfa.uji.es glycol (DEG), a known poison used in antifreezes and as a solvent [1]. Both glycerine and diethylene glycol are similar in appearance, smell and taste. This followed an incident with dozens of hospitalizations for serious intoxication and more than 100 confirmed deaths in Panama in September 2006. According to information published by the New York Times, this resulted from a Chinese factory deliberately falsifying records in order to export the cheaper DEG as the more expensive glycerol for use in cough syrup preparation [2]. In the 1990s several incidents were reported concerning DEG-contaminated glycerine in acetaminophen syrup in different countries, which resulted in hundreds of deaths. Toxic syrup has figured in at least eight mass poisonings around the world in the past two decades, with researchers estimating that thousands have died. In fact, the first references of poisonings by DEG date back to 1937, when more than 100 people, many of them children, died in the US after ingesting DEG-contaminated Elixir Sulfanilamide, a drug used to treat infections. This tragedy led to tough drug regulations and the start of the modern FDA [2].

Over the years, DEG has been loaded into all varieties of medicine: cough syrup, fever medication, injectable drugs, etc. In one of the last incidents (May 2007), federal health officials discovered that DEG-contaminated Chinese toothpaste had entered the US and been distributed in hospitals for the mentally ill, prisons, juvenile detention centres and some hospitals [3]. The European Union also warned about the entry of this product into Spain, which might have been distributed in hotels and hospitals. This generated high public concern, as revealed by the news published in the most-read newspapers. DEG contents of above $5 \%$ were reported in some samples, and thousands of illegal toothpaste tubes were removed by national health authorities [4]. 
In July 2007, our laboratory was asked by the health department of our regional government (Generalitat Valenciana) to analyse toothpaste tubes samples suspected of being contaminated with DEG that were distributed to several hospitals in the Valencia region [5].

Due to the concern over DEG poisoning, reliable analytical methods to detect, identify and quantify this compound are required. The FDA applies the method of Kenyon et al. [6], based on thin-layer chromatography (TLC) as an initial screening to detect the presence of DEG in toothpaste at a level of $0.1 \%$ weight $(1000 \mathrm{mg} / \mathrm{kg})$. Once a positive result for the presence of DEG is found, a second procedure based on gas chromatography-mass spectrometry (GC-MS) in full-scan mode is applied for confirmation and a more accurate estimation [7]. This method can be used for the determination of diethylene glycol and ethylene glycol in toothpaste at levels of $1000 \mathrm{mg} / \mathrm{kg}(0.1 \%$ by weight $)$ and above. However, identity confirmation may be problematic, because there are few unique ions among these analytes and, as a consequence, the prospect of significant interferences exists. The same authors reference an approach that could be used for extra confirmation, which consists of preparing silyl derivatives of the analytes for GC-MS determination under different conditions [8].

The determination of DEG is difficult due to its high polarity, low volatility, low mass and lack of chemical groups that could facilitate its detection. In GC-MS analysis, there can be many potential interferences at the low masses where abundant ions are observed, whereas the sensitivity for higher mass ions, although more useful from a qualitative point of view, is relatively poor. As was mentioned above, an alternative involving the preparation of silyl derivatives has been applied. However, derivatization procedures are normally time-consuming and require more sample manipulation.

The physicochemical characteristics of this compound fit better with LC analysis, although a lack of adequate chemical groups (e.g. chromophores, fluorophores) hampers its measurement by conventional detectors. For these reasons, MS appears to be the ideal technique for determining this compound. In order to obtain enough sensitivity and selectivity, MS/MS methods are normally preferred. However, due to the low molecular weight of DEG, it is difficult to obtain adequate product ions.

Due to recent advances in mass spectrometry, the orthogonal accelerated time-of-flight mass analyzer (TOF) and hybrid quadrupole TOF-MS (QTOF) have become more readily available. These instruments have added higher MS resolution over traditional quadrupole, triple quadrupole or ion trap MS analyzers (resolutions of $10,000-17,500$ can typically be obtained), which provides accurate mass data and enables confident identification of the compounds detected in samples. The low mass errors (typically below $5 \mathrm{ppm}$ ) of modern TOF analyzers and their excellent sensitivities when used in full-scan acquisition mode give them extraordinary potential for identification purposes and for elucidating the structures of unknown chemical contaminants. LC-TOF-MS has been satisfactorily applied in the environmental field $[9,10]$ and in food safety $[11,12]$ and has allowed the elucidation of pesticide metabolites and unknown compounds [13-15]. The potential of this technique for identification purposes and for elucidating the structures of unknown chemical contaminants has been emphasised in detailed reviews recently published in Analytical Chemistry on water analysis [16] and on environmental mass spectrometry [17].

Ultra-performance liquid chromatography (UPLC) uses small-diameter particles (typically $1.7 \mu \mathrm{m}$ ) in the stationary phase and short columns, which lead to narrower LC peaks (5-10 s wide) and improved chromatographic separations, with short analysis times (often $10 \mathrm{~min}$ or less) [16]. Hyphenation of UPLC with TOF-MS is very attractive due to the improved analytical characteristics resulting from both techniques. However, only a few examples of applications of this technique can be found in the literature, due to the very recent introduction of this instrumentation in research laboratories $[18,19]$.

In this work, a rapid and efficient method for the rapid screening of DEG in toothpaste based on UPLC-TOF-MS has been developed, which is able to detect, identify and quantify this toxic compound at levels as low as $0.005 \%$ in samples.

\section{Experimental}

\section{Chemicals and solvents}

Diethylene glycol reference standard was purchased from Sigma-Aldrich (St. Louis, MO, USA). HPLC-grade methanol, reagent-grade formic acid ( $\mathrm{HCOOH}$, content $98-100 \%$ ), sodium hydroxide and ammonium acetate $\left(\mathrm{NH}_{4} \mathrm{OAc},>98 \%\right)$ were purchased from Scharlab (Barcelona, Spain). HPLCgrade water was obtained by passing distilled water through a MilliQ water purification system (Millipore, Bedford, MA, USA). Leucine enkephalin, used as the lock mass, and lithium acetate dihydrate $\left(\mathrm{LiOAc} \cdot 2 \mathrm{H}_{2} \mathrm{O},>63 \%\right)$ were purchased from Sigma.

A stock standard solution of diethylene glycol was prepared by dissolving approximately $5 \mathrm{~g}$, accurately weighed, in $10 \mathrm{~mL}$ of HPLC-grade water obtaining a concentration of approximately $500 \mathrm{~g} / \mathrm{L}$. For LC-MS analysis, the stock solution was diluted with water to obtain working solutions. 




(a)



(b)



(c)

Fig. 1 UPLC-TOF-MS chromatograms corresponding to (a) blank toothpaste, (b) matrix-matched standard of diethylene glycol (0.5 $\mu \mathrm{g} / \mathrm{mL})$ and (c) toothpaste sample spiked at $50 \mathrm{mg} / \mathrm{kg}$ (corresponding to $0.5 \mu \mathrm{g} / \mathrm{mL}$ in the final extract)

\section{Instrumentation}

An ultra-performance Waters Acquity liquid chromatography (UPLC ${ }^{\mathrm{TM}}$ ) system (Waters, Milford, MA, USA) was interfaced to a QTOF mass spectrometer (QTOF Premier, Waters Micromass, Manchester, UK) using an orthogonal Z-spray electrospray interface. LC separation was performed using an Acquity UPLC $\mathrm{BEH} \mathrm{C}_{18}$ analytical column (Waters, $2.1 \times 50 \mathrm{~mm}, 1.7 \mu \mathrm{m}$ ) at a flow rate of $300 \mu \mathrm{L} / \mathrm{min}$. The mobile phase used was a timeprogrammed gradient using $\mathrm{H}_{2} \mathrm{O} \mathrm{MeOH}$ (96:4) and $\mathrm{MeOH}$ $(100 \%)$, where the percentage of organic modifier was changed linearly from $0 \%$ to $90 \%$ in $1 \mathrm{~min}$. The injection volume was $10 \mu \mathrm{L}$. TOF-MS resolution was $\sim 10,000 \mathrm{FWHM}$ (V-mode) at $m / z 556$. The MCP detector potential was set to $1750 \mathrm{~V}$ in positive ionisation mode. A cone voltage of $10 \mathrm{~V}$ and a capillary voltage of $3 \mathrm{kV}$ were used. The interface temperature was set to $350{ }^{\circ} \mathrm{C}$ and the source temperature to $120{ }^{\circ} \mathrm{C}$. A scan time of $0.1 \mathrm{~s}$ was chosen. The automated attenuated function (dynamic range enhancement, DRE) was selected to correct possible mass peak saturations, making it feasible to achieve quantification and accurate mass measurements over a wide concentration range. Calibration experiments from 50 to $1000 \mathrm{~m} / \mathrm{z}$ were performed monthly using a mixture of $\mathrm{NaOH} 0.05 \mathrm{M}$ : $\mathrm{HCOOH} 10 \%$ (50:50). A $2 \mathrm{mg} / \mathrm{L}$ standard solution of leucine enkephalin was introduced via the lock spray needle (cone voltage, $90 \mathrm{~V}$ ) at a flow rate of $30 \mu \mathrm{L} / \mathrm{min}$.

\section{Analytical procedure}

Around $1.0 \mathrm{~g}$ of a homogenised toothpaste sample was transferred to centrifuge tubes $(50 \mathrm{~mL})$ and extracted with water $(10 \mathrm{~mL})$ on a mechanical shaker for $30 \mathrm{~min}$. After centrifugation at $3500 \mathrm{rpm}$ for $15 \mathrm{~min}$, the supernatant was diluted tenfold with HPLC-grade water. Finally, $10 \mu \mathrm{L}$ of the diluted extract was injected directly into the UPLCESI-(Q)TOF-MS system in full acquisition mode.

Quantification was performed by using matrix-matched standards prepared in a toothpaste blank extract diluted tenfold (1 g sample diluted in $100 \mathrm{~mL}$ HPLC-grade water).

\section{Results and discussion}

MS optimization

The positive-ion electrospray full-scan spectrum of a DEG reference standard in solvent contained three peaks at $m / z$ 107.0701, 124.0970 and 129.0517 corresponding to the protonated molecule $[\mathrm{M}+\mathrm{H}]^{+}(0.7 \mathrm{mDa})$, and the ammonium $\left[\mathrm{M}+\mathrm{NH}_{4}\right]^{+}(-0.4 \mathrm{mDa})$ and sodium adducts $[\mathrm{M}+\mathrm{Na}]^{+}$ 


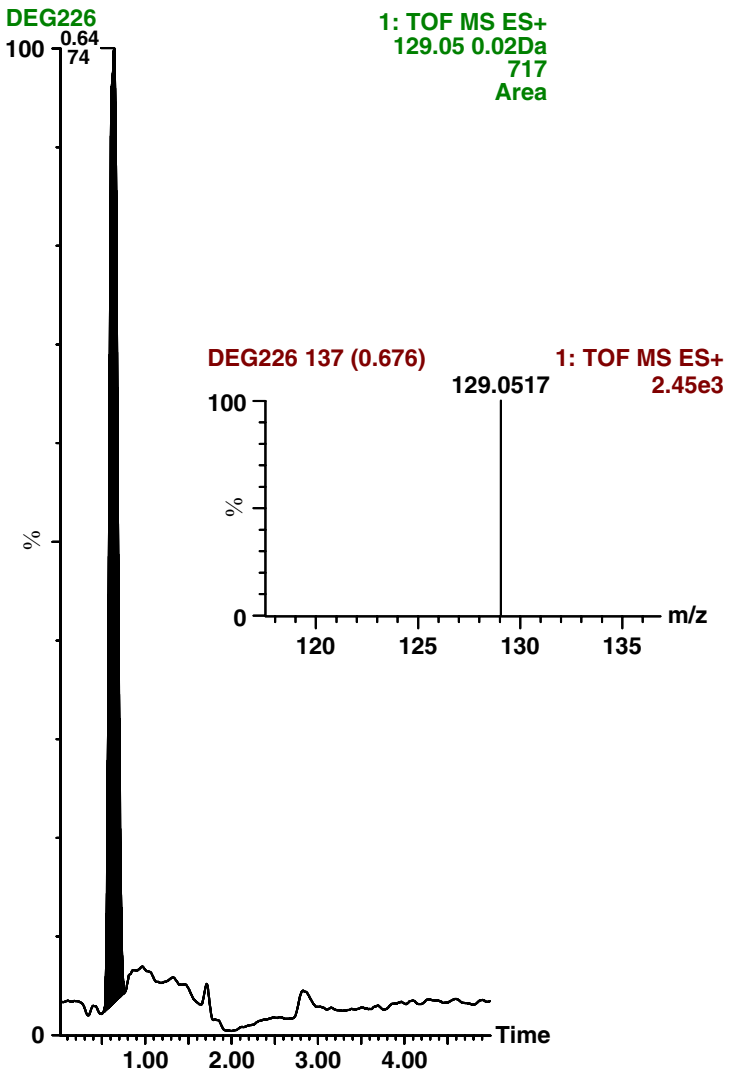

(a)

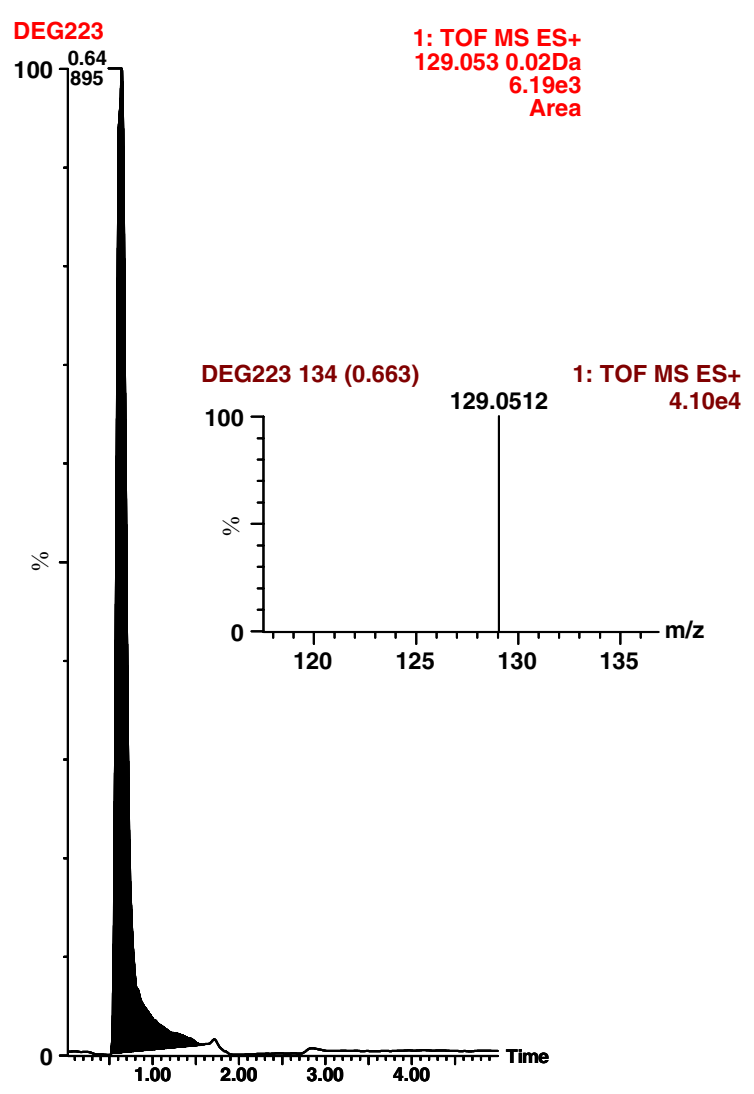

(b)

Fig. 2 UPLC-TOF-MS chromatograms corresponding to toothpaste samples containing (a) diethylene glycol at a concentration level of $<50 \mathrm{mg} /$ $\mathrm{kg}$, and (b) diethylene glycol at a concentration level of $914 \mathrm{mg} / \mathrm{kg}$

$(1.1 \mathrm{mDa})$, respectively. However, after injecting a DEG standard into toothpaste extract, no peak corresponding to the protonated molecule was observed. Instead, the peak at $m / z 129.0525(-0.3 \mathrm{mDa})$ was the only one observed. This is expected, considering the high content of sodium in toothpaste samples.

When MS/MS experiments were performed no fragments were observed in the spectra even when the collision energy was increased. This poor fragmentation of sodium adducts has already been explained in the literature [20]. Although different ways to promote the formation of the protonated molecule or the ammonium or lithium adducts were attempted, like adding formic acid, ammonium acetate or lithium acetate [21] to the mobile phase or to the sample extract, no satisfactory results were obtained. Finally, the DEG sodium adduct was selected for determination in TOF mode.

\section{Method optimisation}

After direct injection of a toothpaste sample extract $(1 \mathrm{~g}$ in $10 \mathrm{~mL}$ water), bad peak shapes and variable retention times were observed. Moreover, when comparing a standard and a blank sample fortified at the same level, a notable difference in the peak intensities was observed. However, no significant difference was found between the blank sample spiked before and after extraction, thus indicating that there were no losses during the extraction step. This difference between the standard and the spiked samples could be due to ionisation suppression from co-extracted components of the matrix. To minimise the matrix overloading and to improve the signal in the sample matrix, a tenfold dilution with water was tested. After diluting the sample extract, a good peak shape and reproducible retention times were obtained. Although the recovery values obtained using external calibration with aqueous standards were satisfactory, matrix-matched calibration (using a blank matrix diluted tenfold) was selected to ensure that there were similar sodium contents in both samples and standards.

\section{Validation}

The optimised method described above was validated before it was applied to real-world samples. Linearity was evaluated by analysing five matrix-matched standard solutions in duplicate, in the range $0.25-25 \mathrm{mg} / \mathrm{L}$; a 


\section{PEG: $\mathrm{HO}-\left[\mathrm{CH}_{2} \mathrm{CH}_{2}-\mathrm{O}\right]$ n- $\mathrm{CH}_{2} \mathrm{CH}_{2} \mathrm{OH}$}

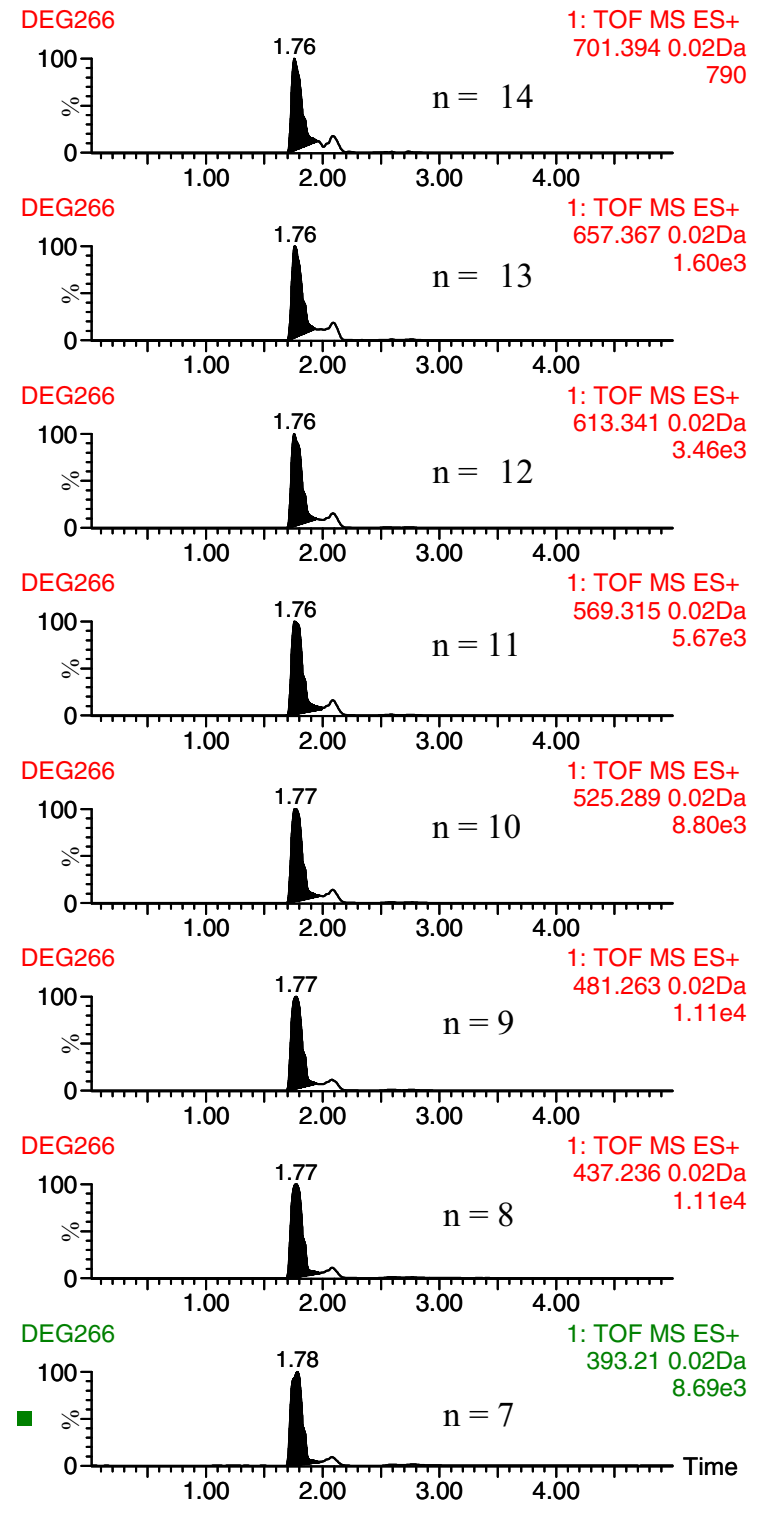

(a)



(b)

Fig. 3 UPLC-TOF-MS chromatograms of two toothpaste samples containing different polyethylene glycols: (a) high molecular weight PEGs and (b) low molecular weight PEGs

quadratic calibration curve with a correlation coefficient $\left(r^{2}\right)$ of higher than 0.995 was obtained.

Within-day precision (repeatability, expressed as relative standard deviation, in \%) and recovery were determined by analysing fortified blank samples in triplicate at three spiking levels: $0.005 \%(50 \mathrm{mg} / \mathrm{kg}), 0.1 \%(1000 \mathrm{mg} / \mathrm{kg})$ and $5 \%(50000 \mathrm{mg} / \mathrm{kg})$. Spiked samples were equilibrated for $1 \mathrm{~h}$ prior to extraction. The method was found to have excellent precision (RSD $<4 \%$ ) and satisfactory accuracy, with recoveries ranging from $74 \%$ to $98 \%$.
The limit of quantification (LOQ) was established as the lowest concentration tested and fully validated in samples with satisfactory recovery $(70-110 \%)$ and precision $(<15 \%$ RSD), and was found to be $50 \mathrm{mg} / \mathrm{kg}(0.005 \%)$.

The limit of detection (LOD), defined as the lowest concentration that the analytical process can reliably differentiate from background levels, was estimated for a signal-to-noise of 3 from the chromatogram at the lowest analyte concentration assayed (i.e. $50 \mathrm{mg} / \mathrm{kg}$ ), and was found to be $8 \mathrm{mg} / \mathrm{kg}$. 
The specificity of the method was evaluated using a blank procedure, a processed blank sample, and a blank sample spiked at the lowest fortification level assayed (LOQ). Under these conditions, the response obtained for both the blank procedure and the blank sample did not exceed $30 \%$ of the response corresponding to the LOQ.

As an example of the excellent sensitivity and selectivity of the method, Fig. 1 shows typical UPLC-TOF-MS chromatograms for the sample used as a blank, a matrixmatched standard solution $(0.5 \mu \mathrm{g} / \mathrm{mL})$ and a toothpaste sample spiked at $0.005 \%(50 \mathrm{mg} / \mathrm{kg})$.

\section{Application to real samples}

The developed UPLC-TOF-MS method was applied to the analysis of 21 toothpaste samples. Five of them were provided by the health department (Generalitat Valenciana). The others were purchased in a chemist (five samples), a supermarket (five samples) and a "1-euro shop" (six samples). All samples were analysed in triplicate.

When processing real samples, a matrix-matched standard calibration curve (between 0.25 and $25 \mathrm{mg} / \mathrm{L}$ ) was injected before and after the samples. Blank samples fortified at the LOQ level were used as quality controls (QC) distributed along the batch of samples every four injections. This blank sample had previously been analysed to confirm the absence of the analyte. QC recoveries were in the range of $70-110 \%$ in all cases, demonstrating the robustness of the method along the analytical sequence.

Diethylene glycol was detected in around $40 \%$ of the samples analysed, although concentration levels did not exceed $0.1 \%$ in any case. Positive samples contained between 27 and $914 \mathrm{mg} / \mathrm{kg}$ DEG (between $0.003 \%$ and $0.09 \%$ ). Figure 2 shows illustrative chromatograms corresponding to the two toothpaste samples containing the lowest (Fig. 2a) and the highest (Fig. 2b) levels found for DEG.

Due to the high mass accuracy of the instrument, a narrow $0.02 \mathrm{Da}$ extraction window could be used, which greatly increased the selectivity of the method when compared to other single MS analysers. The accurate mass measurements, together with the use of retention time, were used to confirm the identity of the analyte. All mass errors were below $2 \mathrm{mDa}$, independent of the DEG concentration in the sample. Deviations of lower than $2 \%$ were observed for all retention times. The combination of measurements assured us that the compound detected in the samples was DEG.

The application of UPLC-TOF-MS also allowed the detection of other polyethylene glycols (PEGs) in the toothpaste samples, from tetraethylene glycol to pentadecaethylene glycol, as shown in Fig. 3. This illustrates the potential of this technique for detecting other analytes that might also be investigated in toothpaste samples.

\section{Conclusions}

The combined use of UPLC and TOF-MS has been proven to be an efficient and advanced approach for the identification and quantification of diethylene glycol in a complex matrix like toothpaste with minimum sample treatment and chromatographic run times of only five minutes. The application of this hyphenated technique to toothpaste samples has resulted in the detection of diethylene glycol in several samples at concentrations of below $0.1 \%$, as well as other substantially larger glycols. Mass errors were $<2 \mathrm{mDa}$ and retention time deviations were $<2 \%$ in all samples analysed.

The elevated mass resolution, the high sensitivity in fullscan mode and the excellent mass accuracy of TOF-MS make this instrument a powerful analytical tool for rapid screening purposes. UPLC-TOF-MS will surely become an essential advanced analytical tool for screening and confirming organic pollutants in the near future.

Acknowledgements The authors are very grateful to the Serveis Centrals d'Instrumentació Científica (SCIC) of University Jaume I for the use of UPLC-QTOF MS (QTOF Premier) and to Generalitat Valenciana for providing some samples suspected to be contaminated with diethylene glycol. The authors acknowledge the financial support from the Ministerio de Educación y Ciencia, Project: CTQ 2006-07594.

\section{References}

1. US FDA (2007) FDA advises manufacturers to test glycerin for possible contamination. US Food and Drug Administration, Rockville, MD

2. Bogdanich W, Hooker J (2007) From China to Panama, a trail of poisoned medicine. New York Times, 6 May 2007

3. Bogdanich W (2007) Wider sale is seen for toothpaste tainted in China. New York Times, 28 June 2007

4. de Cózar A (2007) La toxicidad de la pasta contaminada es superior a la detectada en EEUU. El País, 7 July 2007

5. Navarro S, Sauquillo MR, Linde P, Zafra I, Gutiérrez J, Pardillas JM (2007) El Hospital de Alicante retira centenares de tubos de dentífricos irregulares. El País, 5 July 2007

6. Kenyon AS, Shi X, Wang Y, Ng WH, Prestridge R, Sharp K (1998) J AOAC Int 81:44-50

7. Litzau J, Mulligan K (2007) GC-MS screening procedure for the presence of diethylene glycol and ethylene glycol in toothpaste, v. 2.0. US FDA, Rockville, MD. http://www.cfsan.fda.gov/ dms/ degmeth.html. Accessed 31 March 2007

8. Mulligan KJ (1996) No. 4042: a procedure to determine diethylene glycol (2,2'-oxybisethanol) and ethylene glycol (1,2-ethanediol) in glycerin and selected products. DHHS Lab Bull 12(7)

9. Sancho JV, Pozo OJ, Ibáñez M, Hernández F (2006) Anal Bioanal Chem 386:987-997

10. Hernández F, Ibáñez M, Pozo OJ, Sancho JV (2004) Anal Chem 76:4349-4357

11. Grimalt S, Pozo OJ, Sancho JV, Hernández F (2007) Anal Chem 79:2833-2843

12. Thurman EM, Ferrer I, Fernández-Alba AR (2005) J Chromatogr A $1067: 127-134$ 
13. Thurman EM, Ferrer I, Malato O, Fernández-Alba AR (2006) Food Addit Contam 23:1169-1178

14. Grange AH, Zumwalt MC, Sovocool GW (2006) Rapid Commun Mass Spectrom 20:89-102

15. Ibáñez M, Sancho JV, Pozo OJ, Niessen WMA, Hernández F (2005) Rapid Commun Mass Spectrom 19:169-178

16. Richardson SD (2007) Anal Chem 79:4295-4324

17. Richardson SD (2006) Anal Chem 78:4021-4046
18. Petrovic M, Gros M, Barcelo D (2006) J Chromatogr A 1124:68-81

19. Ibáñez M, McMillan D, Sancho JV, Rao R, Hernández F (2008) Trends Anal Chem (in press)

20. Fernández-Puente P, Fidalgo-Sáez MJ, Hamilton B, Furey A, James KJ (2004) Toxicon 44:919-926

21. Grimalt S, Pozo OJ, Marín JM, Sancho JV, Hernández F (2005) J Am Soc Mass Spectrom 16:1619-1630 